\title{
Universal CAR T cell: engineering of universal T cell, modular CAR system, and applications
}

\author{
Zhipu Duan ${ }^{1,}{ }^{*}, \dagger$, Zhuohui Lin ${ }^{2,}{ }^{*}$, , Shijie Zhou ${ }^{3,}{ }^{*}, \dagger$ \\ ${ }^{1}$ Department of Chemistry and Biochemistry University of California Los Angeles Los Angeles, California, \\ America \\ ${ }^{2}$ Faculty of Microbiology and Immunology University of British Columbia Vancouver, British Columbia, \\ Canada \\ ${ }^{3}$ Department of Nursing John Hopkins Hospital Baltimore, Maryland, America \\ *Corresponding author: zhipu007@g.ucla.edu, zlin11@student.ubc.ca, szhou63@jhmi.edu \\ "These authors contributed equally.
}

Keywords: Universal Chimeric Antigen Receptor $\mathrm{T}$ cells(CAR T), graft-versus-host-disease (GVHD), Immunogenicity

\begin{abstract}
Universal Chimeric Antigen Receptor T cells (CAR T), an alternative design based on conventional CAR T cells, uses a switchable adaptor for a better redirection towards the target site. This technology overcomes the obstacles of the conventional Car $\mathrm{T}$ cells system, such as immunogenicity, massive expression of cytokine and fixed antigen specificity. This article introduces universal CAR $\mathrm{T}$ cells from both the perspectives of the universal $\mathrm{T}$ cells and its modular CAR systems, illustrating the advancement of universal CAR $\mathrm{T}$ cells to overcome the limitation of conventional CAR $\mathrm{T}$ cells and serve as a more controllable and highly promising system. The universal CAR $\mathrm{T}$ cells section focuses on the challenges of choosing $\mathrm{T}$ cell sources and the corresponding solutions, while the modular CAR system section summarizes the different types of switchable adaptors in combination with clinical applications in various types of cancer treatments. Overall, universal CAR T cells therapy is a novel development that not only out-competes but also recovers the shortage of the conventional CAR T cells system. With the use of switchable adaptors, the universal CAR T cells system is commercially beneficial for the public and a safe product to allow the industry to expand the clinical application of different types of cancers.
\end{abstract}

\section{Introduction}

Chimeric Antigen Receptor (CAR) T cell therapy has been demonstrated as a potent anti-cancer therapeutic. The conventional CAR T cell consists of an extracellular targeting domain, single-chain antibody variable fragment (scFv) connected to an intracellular signalling domain. The scFv targets antigen on the tumor cancer and activates the $\mathrm{T}$ cell effector activity through the signalling domain, leading to the lysis of the target tumor cells [1]. Conventional CAR T cell therapy has been demonstrated with promising results in clinical trials, and several CAR T cell therapies have been approved by the U.S. Food and Drug Administration (FDA) [2].

Regardless of the clinical advances, conventional CAR T cell therapy still faces challenges and limitations, preventing it from being applied to a large-scale clinical. First, conventional CAR T-cell therapy is generated from an autologous (patient itself) $\mathrm{T}$ cells production platform, which needs to be prepared case-by-case on a custom-made basis. Therefore, it is time-consuming and expensive. The average waiting period for autologous CAR T cells is between 2 to 4 weeks [3, 4]. Also, the present manufacturing line has a high risk of production failure, which accounts for 5-13\% of the cases [5]. Second, since conventional CAR T cell therapy relies on autologous $\mathrm{T}$ cell sources, the quantity and the quality of the patient's $\mathrm{T}$ cell limits the efficacy of the products. It is not always possible to manufacture an effective therapeutic product in patients with immunodeficiency. Heavily treated 
cancer patients, especially infants, are also subjected to this limitation [6]. Third, the conventional CAR T cells also have a fixed targeting specificity and are vulnerable to antigen-loss relapse caused by downregulation of the targeting antigen. There have been many reported cases of antigen-negative relapse after CD19 CAR T therapy in clinical trials [7]. Lastly, conventional CAR T cell therapies have the risk of side effects. Conventional CAR T cells might non-specifically target healthy tissues and cause massive cytokine release [2]. Conventional CAR T cell therapies require careful monitoring of the side effects and preparation for mitigation.

The idea of developing universal CAR T cell therapies is to overcome these challenges and develop a more accessible and efficient CAR T cell treatment. The current research on universal CAR T cells could be divided into two areas: universal T cells and modular CAR systems. The development of universal $\mathrm{T}$ cells focuses on developing ready-to-use off-the-shelf universal allogeneic $\mathrm{T}$ cell or CAR $\mathrm{T}$ cell products. This will overcome the limitations of manufacturing conventional CAR T cell therapies and make them more accessible and beneficial to a larger population. On the other hand, the development of modular CAR systems aims to design a CAR system that could be easily redirected to targeting different tumor-associated antigens (TAA). This will offer better flexibility, controllability, and safety compared to conventional CAR T cell therapies.

Overall, universal CAR T cells can efficiently target different types of cancers such as infant B-cell acute lymphoblastic leukemia, hepatocellular carcinoma, acute myeloid leukemia with the use of different modulators. Such a system can precisely target the cancer cell receptors, lowering the risk of graft vs. host disease, cytokine release syndrome (CRS), and the potential of antigen loss. Thus, the universal CAR T cell system is more pervasive to patients and is used to target cancers that conventional CAR T cell therapy cannot treat cancers.

\section{Engineering of universal $T$ cells}

Conventional CAR T cell therapy using autologous $\mathrm{T}$ cell sources has many limitations, including huge time and economic expanse and risk of failure [3-5]. Using autologous $\mathrm{T}$ cell sources makes it impossible for patients who cannot provide robust $\mathrm{T}$ cells [6]. Using donor-derived allogeneic $\mathrm{T}$ cells to produce CAR T cell products will overcome these limitations and offer ready-to-use off-the-shelf CAR T cell products that benefit a larger patient population.

\subsection{Challenges and Strategies of Engineering of universal $T$ cells}

The allogeneic CAR T cell is a beautiful idea. However, high alloreactive characteristics of T cells make adoptive transferring allogeneic $\mathrm{T}$ cells face an increased risk of rejection between donor and host. The infusion of allogeneic $T$ cells may cause graft-versus-host-disease (GVHD) and immunogenicity.

\subsubsection{Graft-versus-host-disease (GVHD)}

Graft-versus-host-disease (GVHD) related to infusion allogeneic CAR T-cell is donor T cells attacking the recipient who causes host organ or tissue damage [8]. When the attack happens, donor $\mathrm{T}$ cells trigger host cell death through FAS ligand, perforin, and granzyme signalling pathways, which may cause fatal host healthy organ and tissue death [9]. When severe GVHD happens, it could be fetal.

\subsubsection{Immunogenicity}

Immunogenicity is another issue caused by host cells rejecting transferred allogeneic CAR T cells due to "foreign" proteins presented on CARs and T cells. As a result, CAR T cells couldn't expand and persist in the host, leading to malignant relapse. Even with lymphodepletion chemotherapy before infusion, delayed rejection of the adoptively transferred $\mathrm{T}$ cells could cause disease relapse. Alloimmunization is another complicated situation accompanied with immunogenicity post allogeneic CAR $\mathrm{T}$ cells transfusion when the recipient develops donor-specific anti-HLA antibodies (DSAs). 
DSAs mean primary graft failure. This may also prevent the patient from getting the same therapy again [10].

\subsection{Strategies to overcome GVHD and immunogenicity}

Both GVHD and immunogenicity would cause T cell grafting failure and be fatal to the recipient. Therefore, mitigating the immunologic mismatch between donor $\mathrm{T}$ cells and recipient immunity system or inhibition of the alloreactivity of donor $\mathrm{T}$ cells is the crucial challenge around engineering a universal allogeneic $\mathrm{T}$ cells production platform. The goal of tackling these issues is that the receiving host immune system won't eliminate new T cells. Instead, it accepts those therapeutic T cells to fight against cancer cells without causing attacks toward healthy organs and tissue. T cell-subset selection and gene editing approaches are two main directions that researchers have been working on.

\subsubsection{T cell-subset selection}

Many T cell subsets have been studied for a long time and have been chosen to solve GVHD. The selection mainly focuses on $\gamma \delta \mathrm{T}$ cells, virus-specific T cells (VSTs), memory T cells, and HCT (Hematopoietic cell transplantation)-donor-derived CAR T cells. According to different chains on T cell receptors (TCR), there are two big groups of T cell subunits: $\alpha \beta$ T cells and $\gamma \delta$ T cells. $\alpha \beta$ T cells are MHC-dependent T cells to recognize foreign or non-self-antigen presenting cells, whereas $\gamma \delta \mathrm{T}$ cells are MHC-independent. The $\alpha \beta$ TCR on donor T cells identifies host antigens as "not-self" when human leukocyte antigen (HLA) mismatches between them, and GVHD happens [11]. Studies on $\gamma \delta$ T cells have shown that these two subsets do not induce GVHD [12]. Virus-specific T cells (VSTs) have been studied for over a decade. Using CAR-transduced allogeneic VSTs has demonstrated as a safe and effective allogeneic platform targeting a variety of TAAs, including CD30, HER2, and GD2, with minimal GVHD risk [11].

Memory T cells subsets also attract researchers' attention as one of the candidates to generate allogeneic CAR T cells due to their lower risk of GVHD in the HLA-mismatched situation. Memory $\mathrm{T}$ cells, in general, are a group of more matured $\mathrm{T}$ cells; therefore, they are less alloreactive than naive $\mathrm{T}$ cells [13]. Besides that, memory T cells also demonstrated enhanced effectiveness and persistence in autologous CAR T-cell platforms. The use of memory $\mathrm{T}$ cell subsets as effector T cells in CAR Tcell therapy is actively studied in autologous settings. In allogeneic settings, the studies are mainly in pre-clinical models. However, CD45RA-negative T cells, including central memory and effector memory $\mathrm{T}$ cell subsets, have been evaluated in human allogeneic HCT and demonstrated a decreased risk of GVHD and feasibility.

HCT-donor-derived CAR T cells is a strategy that Brudno and his team have adopted to treat patients with progressive B-cell malignancies after transplant. The team used allogeneic CD19directed CAR T cells derived from an individual patient's hematopoietic stem-cell transplantation (alloHSCT) donor and demonstrated anti-tumour benefits and safety, including no new-onset GVHD [14].

\subsubsection{Gene editing approaches}

Gene editing technologies are hotly evaluated to generate allogeneic T cells that would not cause GVHD and immunogenicity. Three mainly studied genome-editing technologies are ZFN (Zinc finger nuclease), TALEN (transcription activator-like effector nuclease), and CRISPR-Cas9. They are used primarily to genetically modify allogeneic TCR constant a chain (TRAC) or $\beta$ chain (TRBC) on ab T cells. Torikai et al. in 2012 used ZFN mediated ablation of TRAC or TRBC. By using the same approach, after removal of specific TCR chains, these T cells still show similar ability to target CD19positive targets, but at the same time didn't cause GVHD [15].

TCR ablation by gene-editing methods such as ZFN, TALEN, and CRISPR/Cas9 may help eliminate GVHD but cannot stop immunogenicity from happening. Researchers found a component of HLA-I protein, b-2 macroglobulin (B2M), present on all $\mathrm{T}$ cells related to immunogenicity. Knockout of the B2M gene from $\mathrm{T}$ cells demonstrated reduced alloreactivity in vivo by Ren et al. [16]. 
CRISPR/Cas9 has been used to successfully knockout TCR and HLA class I (b2-microglobulin, B2M). Hu et al. have evaluated a universal CD19/CD22 dual-targeting allogeneic CAR T-cell product (CTA101) with CRISPR-edited TRAC region, and CD52 gene knocked out on relapsed/refractory Bcell acute lymphoblastic leukemia (r/r ALL). CD19- relapse happened to 39\% of patients who have received conventional autologous CD19 targeted CAR-T therapy [17]. During this clinical trial, the open-label dose-escalation phase I study successfully proved to avoid host immune-mediated rejection and support alemtuzumab (anti-CD52 antibody)-mediated depletion of $\mathrm{T}$ cells. In addition, this CD19/CD22 dual-targeting allogeneic CAR T-cell product demonstrated potent anti-tumor activity and persistence and addressed CD19 escape this common cause of disease relapse. With its more straightforward design, improved editing efficiency, feasibility, and most importantly, no doselimiting toxicities (DLTs), GVHD, or neurotoxicity syndrome occurred, CRISPR/Cas9 has shown potent potential and safer profile, shorter culture time compared with TALEN, and better feasibility [18].

This is the first CRISPR-edited allogeneic CD19/CD22 dual-targeted CAR T cells product to achieve CR in pts with B-ALL. All patients who have responded showed minimal residual disease (MRD)-negative remission on day 28 post-infusion. This ground-breaking evaluation has also demonstrated the potential that dual-targeted CAR T cells may prevent single-target loss-induced relapse [19].

\section{Universal Modular CAR system}

Besides engineering universal T cells, researchers also focus on modulating the CAR receptor. The conventional CAR has a fixed targeting specificity, therefore, is susceptible to antigen-loss relapses caused by the immunoediting of the tumor cells [20]. Redirecting conventional CAR T cells to combat antigen loss requires additional genetic modification, labour-intensive and time-consuming. A universal modular CAR system has been developed to overcome these challenges, which can be easily redirected without further genetic modification.

Table 1: separable linkers that have been developed in the past.

\begin{tabular}{|c|c|}
\hline Name & Switch elements \\
\hline $\begin{array}{c}\text { Biotin-Binding Immune Receptor } \\
\text { (BBIR) }\end{array}$ & Biotin+dimeric avidin [21] \\
\hline $\begin{array}{c}\text { Anti Fluorescein isothiocyanate } \\
\text { (FITC) CAR }\end{array}$ & fluorescein isothiocyanate (FITC)+anti FITC scFv [22] \\
\hline $\begin{array}{c}\text { SpyTag-SpyCatcher Universal } \\
\text { CAR }\end{array}$ & Fibronectin-binding protein from Streptococcus pyogenes [23] \\
\hline $\begin{array}{c}\text { Split, universal, and programmable } \\
\text { (SUPRA) CAR }\end{array}$ & \begin{tabular}{c} 
Leucine zipper [24] \\
\hline ConvertibleCAR strategy \\
SNAP CAR strategy
\end{tabular} \\
\hline $\begin{array}{c}\text { Co-Localization-Dependent } \\
\text { Protein Switch (Co-LOCKR) CAR }\end{array}$ & inertNKG2D and orthogonal ligand U2S3 [25] \\
\hline UniCAR & anti-La protein scFv and peptide motif of ten aa (5B9 tag) [28] \\
\hline Anti-PNE CAR & 14 aa peptide neo-epitope (PNE) and anti-PNE scFv [29] \\
\hline
\end{tabular}




\subsection{Modular CAR systems' Structure}

The simple redirection is achieved through a splitable design of the modular CAR system (Fig 1). The conventional CAR consists of a targeting domain for recognizing and binding with target tumor cells and an intracellular T cell signalling domain for activating the killing activities of T cells [1]. The conventional CAR uses a fixed connection between the two functional domains. In contrast, the modular CAR structure uses a separable linker. The targeting domain is tagged with a switch element that strongly interacts with a specific receptor. The receptor is connected to the intracellular signalling domain as a signalling module. The tagged targeting domain acts as a switchable adaptor element, and the signalling module is integrated into the modular CAR T cell main body. When competent, the adaptor elements are connected to the signalling module through the interaction between the switch element and the receptor [30]. The connection forms an integral and functional CAR structure and allows the $\mathrm{T}$ cell to activate its killing activities.

Figure 1: This demonstrates the structural difference between the conventional CAR and modular CAR system. The significant difference is the split design of the modular CAR system [30]. (A) Conventional CAR structure consists of scFv targeting TAAs and T cell signalling domain to activate the anti-tumor activities. The connection between these two functional domains is rigid. (B) The modular CAR system has a split design between the targeting domain and the signalling domain. The TAA targeting domain is connected with a switch element to form an adaptor. The signalling module is linked to a component that could bind with the switch element to form an integral active CAR structure and activate the T-cell anti-tumor activities.

\subsection{Choices of Switch elements and different characteristics}

Most of the modular CAR systems share a similar structure. The difference is the choice of switch elements. Over the past, researchers have developed various linkers to modulate the CAR system (Table 1). All the switch elements fulfill the function to connect the targeting elements adaptor and the signalling module on the modular CAR T cell. Different switch elements have slightly different characteristics. These differences mainly affect the administration and immunogenicity of the modular CAR system.

The choice of switch elements affects the administration method of the modular CAR T cell. The method of administration depends on the strength of the connection between the switch elements and the signalling module. A weaker and transient connection requires the adaptor targeting elements and the CAR T cell to be co-delivered. In contrast, a more robust connection allows the modular CAR T cells to be pre-armed with adaptors. Most modular CAR systems have a transient interaction, and the adaptor and the CAR T cells have to be co-administered to achieve anti-tumor functions. The BBIR system has been tested to be loaded with adaptors prior to administration, and the binding is insufficient to last [31]. On the other hand, the SpyTag-SpyCather CAR system and the SNAP CAR system have a more substantial and long-lasting interaction, therefore, could be pre-armed before administration [32] [26]. The difference in administration, in general, does not affect the modular CAR system's flexibility and controllability.

The different switch elements also have a different risk of inducing immune responses in the patient. Some switch elements are more likely to be recognized by the receiving patient's immune system and being attacked by the host immune system. The potential of causing immune response depends on the origin of the switch elements. For example, the SpyTag/SpyCatcher CAR system utilizes bacteriaoriginated fibronectin-binding protein as the switch element. This bacterial origin increases the risk of recognition by the patient's immune system [32]. In contrast, the SNAP-tag used in SNAP CAR has a human origin, therefore, is less likely to be immunogenic [26]. Immunogenicity would be an essential parameter to consider when designing the modular CAR system. 


\subsection{Advantages of modular CAR system over conventional CAR T cells}

\subsubsection{Overcome antigen loss relapse and heterogeneous tumor population}

One of the significant advantages of the modular CAR system is that the modular CAR system can be redirected without the need for genetic modification. This can be used to fight the antigen-loss relapse due to tumor immunoediting. When down-regulation of targeting antigen happens, modular CAR systems could be redirected by switching the targeting elements to eliminate the antigen-loss tumor cells and prevent the relapse.

The modular CAR T cell could also target multiple TAA to combat the antigen-loss relapse and heterogeneous tumor populations. Conventional CAR T cells with single fixed targeting specificity likely result in tumor escaped variants and lead to antigen loss relapses. In the case of heterogeneous tumors, there might not be a shared antigen that presents in all tumor cells. In both cases, the use of CAR T cells targeting multiple antigens could overcome the problem. For conventional CAR T cells, this further increases the burden on time and expense on manufacture. In contrast, multi-targeting could be achieved through a modular CAR system by co-culturing modular CAR T cells with multiple different adaptors or co-delivery of various adaptors. Previous research has effectively reduced the risk of tumor escape variants using bispecific targeting modular CAR T cells [28].

\subsubsection{Expand the use of targeting elements}

The split design of the modular CAR system also offers more flexibility on the use of TAA targeting elements. The conventional CAR mostly utilizes single-chain variable fragments (scFv) as the targeting elements. The targeting elements are mostly antibody derivatives. For the modular CAR systems, TAA targeting elements that could be tagged with a suitable switch element has the potential to be a working adaptor. Researchers have demonstrated the use of antigen-binding fragments (Fabs), nanobodies, and full-length immunoglobulin (IgG) in modular CAR systems [30]. Small biomolecules like folate have also been demonstrated to be incorporated into modular CAR systems targeting unconventional biomarkers [33].

A modular CAR system has also been demonstrated to restore the usefulness of some monoclonal antibodies. In a study by Tamada et al., a modular CAR system with fluorescein isothiocyanate (FITC) as switch elements utilize monoclonal antibody cetuximab as the TAA targeting elements. Cetuximab had been previously demonstrated to have no therapeutic benefits in patients with Kras mutations. Surprisingly, anti-tumor effects were shown when using cetuximab in the modular CAR T cells [22].

\subsubsection{Enhanced treatment safety}

The modular CAR system also provides better safety management compared to conventional CAR $\mathrm{T}$ cells. The conventional CAR T cell is susceptible to the problem of off-tumor on-target side effects, which is CAR T cell attacking non-cancerous tissue. Also, CAR T cell therapy sometimes leads to an unnecessary significant release of cytokine, cytokine release syndrome [2]. Conventional CAR T cell therapy requires careful supervision on the side effects. The modular CAR system offers more options to mitigate the side effects.

The modular CAR T system has a dose-dependent anti-tumor activity. Most modular CAR T cells are co-delivered to the target tumor site to exert the anti-tumor function. The modular CAR T cell only activates the killing mechanism with the present adaptors' elements. The anti-tumor activity depends on the quantity of the adaptor administered. The interaction with the adaptor is not permanent for most modular CAR systems; therefore, a constant supply of adaptor elements is required to keep the antitumor effects. This allows the killing activity to be controlled and halted at any time. Small biomolecules with short half-life could be used as adaptor elements for some modular CAR systems. A shorter half-life further reduces the risk of side effects and would be out of non-cancerous tissue faster [34].

The interaction between the adaptor elements and the signalling module on the modular CAR T cell could be inhibited with competitive inhibitors as a safety off-switch. Competitive inhibitors could interfere with the interaction between the adaptor and the signalling module through binding with 
either adaptor or the signal module with stronger affinity. In some of the modular CAR systems developed, successful interruption of the T cell activity was demonstrated using inhibitors [35].

Some of the modular CAR systems could also be adjusted to reach the killing efficacy of the desired intensity. Cho and her team developed a split, universal, and programmable (SUPRA) CAR system that used a leucine zipper as the switch element. Cho and her team adjusted the affinity between the two leucine zipper components, between the targeting element and leucine zipper, between the signalling module and leucine zipper. They demonstrated a corresponding change in the killing efficacy based on the change they made. They further demonstrate the potential for the modular CAR T cell to be modified to fit the safety concerns [36]. However, this kind of flexibility is not investigated in other modular CAR systems that have been discovered.

The modular CAR T cells could also be programmed with AND, OR, and NOT logic gates for precision killing. This enhances the preciseness of anti-tumor effect and prevents on-target off-tumor side effects. Lajoie et al. developed a modular CAR system with a linker protein switch called colocalization-dependent protein switch (Co-LOCKR). He and his team could program the modular CAR T cell to preferentially kill tumor cells with the specific combination of antigens over the other combinations [37].

\section{Universal CAR T cell real case studies}

Previously, we have discussed the progress on developing the universal CAR T cells and the advantages of universal CAR T cells over conventional CAR T cells. Here we present three studies conducted by Qasim et al., Xuan et al., and Cartellieri et al. on universal CAR T cells against particular cancer. Their studies demonstrated the versatile and advantageous nature of the universal CAR T cell.

\subsection{Universal CAR T cell treating infant B-cell acute lymphoblastic leukemia (B-ALL)}

In this study, a universal allogeneic CAR T cell was developed and used to treat two infants with B-cell acute lymphoblastic leukemia (B-ALL). The two lymphogenic infants are not feasible to manufacture autologous CAR T cells due to heavy cancer treatment. The two infants had relapsed refractory CD19 positive B-ALL and had received lymphodepleting chemotherapy and anti-CD52 serotherapy. Single-dose universal anti-CD19 CAR T was administrated to the and achieved molecular in both infants within 28 days [38]. The universal CAR T cell therapy served as a safeguard before the allogeneic stem cell transplantation. Both infants fully recovered after the transplantation [38]. The study demonstrated the therapeutic potential of universal CAR T cell therapy and its advantage in overcoming the limitation of producing autologous CAR T cells.

The universal allogeneic CAR T cell was engineered through gene-editing to disrupt the expression of CD52 and a $\beta$ T cell receptor (TCRa $\beta$ ). The editing was conducted through transcription activatorlike effector nuclease (TALEN) to disrupt the gene coding regions of CD52 and TCRa $\beta$. The removal of CD52 prevented the universal CAR T cell from being targeted by an anti-CD52 lymphodepletion antibody. The disruption of TCRa $\beta$ aimed to minimize the risk of GVHD [38]. The gene-editing allows the allogeneic CAR T cell to be able to persist inpatient without causing GVHD or immunogenicity. Only mild skin GVHD was reported in one of the infants three weeks after infusion [38]. Overall, the study demonstrated an effective and safe universal allogeneic CAR T cell treatment applied to patients who were not feasible to autologous CAR T cell therapy.

\subsection{Modular CAR system targeting Hepatocellular carcinoma (HCC)}

Xuan et al. have developed a modular CAR T cell to target human glypican-3 (hGPC3) to treat Hepatocellular carcinoma (HCC) [39]. In this study, the universal modular CAR T cell has been demonstrated to have a comparable anti-tumour effect while, more importantly, having a better safety control over conventional CAR T cell therapies. As the fifth most commonly occurring cancer, Hepatocellular carcinoma has limited effective treatment options [39]. Previous studies have demonstrated that hGPC3 has a high expression in HCC and a limited expression in normal tissues, which makes it an ideal target for immunotherapy [40]. Several hGPC3-targeting CAR T cell therapies 
have been developed and demonstrated as efficient [41]. However, the conventional CAR T cells therapies are subjected to the risk of cytokine release syndrome (CRS). In order to improve the safety of CAR T cell treatment, Xuan et al. developed a modular CAR system targeting hGPC3 with enhanced controllability to treat HCC and reduce the risk of CRS [39].

This modular CAR system uses Spy-tag as the switch element. The anti-hGPC3 scFv was engineered to link with Spy-tag as the adaptor [39]. SpyTag is a 13 amino acid peptide derived from Streptococcus pyogenes that binds with SpyCatcher simultaneously [42]. SpyCatcher was linked to the signalling domain on the CAR T cell. The adaptor recognizes hGPC3 on the targeting HCC and recruits and interacts with the SpyCatcher of the signalling module on CAR T cell and activates the anti-tumor effects.

Xuan et al. demonstrated the efficacy of the modular CAR T cell using culturing assays and a xenograft mouse model. In vitro results showed that the modular CAR T cell effectively lyses hGPC3 positive HCC from different cell lines. In vivo, the modular CAR T cell could effectively suppress tumor growth. The anti-tumor effect is comparable to conventional CAR T cells [39]. The cytokine releasing of the modular CAR $\mathrm{T}$ cell was also measured and compared to the conventional CAR T cells. The modular CAR $\mathrm{T}$ cell released significantly lower levels of cytokines, therefore demonstrating better safety [39]. The lower cytokine release was achieved through titrating the binding density of the modular CAR T cell with the HCC by changing the amount of adaptor targeting elements [39].

\subsection{Targeting CD33 and CD123 to treat acute myeloid leukemia (AML)}

Cartellieri et al. has developed a modular CAR T cell, UniCAR, targeting both CD33 and CD123 antigens on the acute myeloid leukemia (AML) tumor cell [28]. The studies demonstrate the dual targeting ability of the modular CAR system to help combat antigen-loss relapse. The studies also showed the dose-dependent anti-tumor effects of the modular CAR system, which enhance the safety of the treatment.

Acute myeloid leukemia (AML) is a heterogeneous leukemic disease [28]. Previous studies have discovered the presence of CD33 and CD123 on nearly 100\% of all AML blasts, either in combination or alone [43]. However, both antigens are not ideal targets for immunotherapy because both antigens are also present on hematopoietic stem cells, on progenitor and mature hematopoietic cells of the myeloid lineage and endothelial cells [44]. Conventional CAR T cell therapies targeting CD33 or CD123 are subjected to the risk of impairment of human hematopoiesis if the CAR T cells are present inpatient [45].

Aiming to overcome these limitations, Cartellieri et al. developed a modular CAR system, UniCAR. The system uses a non-immunogenic ten-amino-acid peptide motif, 5B9 tag, as the switch elements. The adaptor consists of CD33 and CD123 targeting elements linked to the 5B9 tag. The signalling domain on the CAR T cell is linked to the anti-La protein that recognizes and interacts with the 5B9 tag on the adaptor [28]. Similar to other modular systems, the adaptor and the UniCAR T cell are codelivered to the targeting tissue. The interaction between the adaptor and the signalling domain activates the anti-tumor effects.

Cartellieri et al. tested the anti-tumor of the CD33 and CD123 dual targeting UniCAR in cultured cell lines. Dual targeting is achieved in two different methods. One method used co-delivery of two monospecific adaptors of CD33 and CD123. The other method used a hybrid bispecific adaptor targeting both CD33 and CD123. The results from the testing demonstrated that the second method is slightly more efficient than the first one. However, both significantly reduce the blasting number and demonstrate practical anti-tumor activities [28]. In the following in vivo testing,

Cartellieri et al. also demonstrated the dose-dependent manner of the UniCAR T cell. The antitumor activities are activated only under the presence of the adaptor. The conventional CAR T cells targeting CD33 or CD123 remain continuously active in inpatient, therefore have risk to impair human hematopoiesis. However, the UniCAR will remain silent inside the patient unless the adaptors are present. By controlling the infusion of the adaptors, impairment on hematopoiesis could be mitigated [28]. 


\section{Conclusion}

Universal CAR T cell system with its switchable adaptor elements is a highly promising therapy that can target different cancers more broadly and conveniently than the conventional CAR T cell. Universal CAR T cell systems overcome the GVHD and immunogenicity that the conventional CAR $\mathrm{T}$ faces, solving them by utilizing $\mathrm{T}$ cell-subset selection and gene editing. Additionally, the universal modular CAR system has shown its high efficiency in clinical application, reducing cytokine release syndrome, antigen loss, and the risk of GVHD. The overall use of the universal CAR T cell system largely benefits a wider range of patients and has a higher efficiency in treating malignant cancers.

From a future perspective, the safety of universal CAR T cell therapy requires further evaluation, especially for universal modular CAR systems. To date, most modular CAR systems have not been tested in human subjects. The potential risk of immunogenicity is not thoroughly investigated. There is limited evaluation of the immunogenicity of the switch elements used in modular CAR systems. Some switch elements that are not human originated are likely subjected to immune response when administered to the patient [39]. Further assessments are necessary for future clinical applications.

There are also limited horizontal comparisons between different switch elements and modular CAR systems. To find one or several best choices of switch element would be beneficial to apply the modular CAR technology to a larger scale. The ultimate goal for the universal CAR T cell product is to be ready-to-use, off-the-shelf, and versatile, being able to target different TAAs as desired. Settling a standard choice of switch elements would help the industry develop different adaptor choices and expand the targeting element repertoire. Imagine patients in the future could use a universal CAR T cell product that could be personalized based on need and have a wide range of adaptor choices available.

\section{References}

[1] Benmebarek, Mohamed-Reda, et al. "Killing mechanisms of chimeric antigen receptor (CAR) T cells." International journal of molecular sciences 20.6 (2019): 1283.

[2] June, Carl H., et al. "CAR T cell immunotherapy for human cancer." Science 359.6382 (2018): 1361-1365.

[3] Buie, Larry W. "Balancing the CAR T: perspectives on efficacy and safety of CAR T-cell therapy in hematologic malignancies." The American Journal of Managed Care 27.13 Suppl (2021): S243S252.

[4] Maude, Shannon L., et al. "Tisagenlecleucel in children and young adults with B-cell lymphoblastic leukemia." New England Journal of Medicine 378.5 (2018): 439-448.

[5] Moreno, E., et al. "Chimeric antigen receptor T cell therapy in oncology-pipeline at a glance: analysis of the clinicaltrials. gov database." Critical Reviews in Oncology/Hematology (2021): 103239.

[6] Grupp, Stephan A., et al. "Chimeric antigen receptor-modified T cells for acute lymphoid leukemia." New England Journal of Medicine 368.16 (2013): 1509-1518.

[7] Park, Jae H., et al. "Long-term follow-up of CD19 CAR therapy in acute lymphoblastic leukemia." New England Journal of Medicine 378.5 (2018): 449-459.

[8] Sanber, Khaled, Bipin Savani, and Tania Jain. "Graft-versus-host disease risk after chimeric antigen receptor T-cell therapy: the diametric opposition of T cells." British journal of haematology (2021).

[9] Benmebarek, Mohamed-Reda, et al. "Killing mechanisms of chimeric antigen receptor (CAR) T cells." International journal of molecular sciences 20.6 (2019): 1283. 
[10] Gladstone, Douglas E., and Maria P. Bettinotti. "HLA donor-specific antibodies in allogeneic hematopoietic stem cell transplantation: challenges and opportunities." Hematology 2014, the American Society of Hematology Education Program Book 2017.1 (2017): 645-650.

[11] Caldwell, Kenneth J., Stephen Gottschalk, and Aimee C. Talleur. "Allogeneic CAR cell therapy—more than a pipe dream." Frontiers in immunology 11 (2021): 3466.

[12] Pauza, C. David, et al. "Gamma delta T cell therapy for cancer: it is good to be local." Frontiers in immunology 9 (2018): 1305.

[13] Anderson, Britt E., et al. "Memory CD4+ T cells do not induce graft-versus-host disease." The Journal of clinical investigation 112.1 (2003): 101-108.

[14] Brudno, Jennifer N., et al. "Allogeneic T cells that express an anti-CD19 chimeric antigen receptor induce remissions of B-cell malignancies that progress after allogeneic hematopoietic stemcell transplantation without causing graft-versus-host disease." Journal of clinical oncology 34.10 (2016): 1112.

[15] Torikai, Hiroki, et al. "A foundation for universal T-cell based immunotherapy: T cells engineered to express a CD19-specific chimeric-antigen-receptor and eliminate expression of endogenous TCR." Blood, The Journal of the American Society of Hematology 119.24 (2012): 56975705.

[16] Ren, Jiangtao, et al. "Multiplex genome editing to generate universal CAR T cells resistant to PD1 inhibition." Clinical cancer research 23.9 (2017): 2255-2266.

[17] Fousek, Kristen, et al. "CAR T-cells that target acute B-lineage leukemia irrespective of CD19 expression." Leukemia 35.1 (2021): 75-89.

[18] Hu, Yongxian, et al. "CRISPR/Cas9-engineered universal CD19/CD22 dual-targeted CAR-T cell therapy for relapsed/refractory B-cell acute lymphoblastic leukemia." Clinical Cancer Research 27.10 (2021): 2764-2772.

[19] Samadani, Ali Akbar, et al. "CAR T-cells profiling in carcinogenesis and tumorigenesis: An overview of CAR T-cells cancer therapy." International Immunopharmacology (2020): 107201.

[20] Xu, Xinjie, et al. "Mechanisms of relapse after CD19 CAR T-cell therapy for acute lymphoblastic leukemia and its prevention and treatment strategies." Frontiers in immunology 10 (2019): 2664.

[21] Urbanska, Katarzyna, et al. "A universal strategy for adoptive immunotherapy of cancer through use of a novel T-cell antigen receptor." Cancer research 72.7 (2012): 1844-1852.

[22] Tamada, Koji, et al. "Redirecting gene-modified T cells toward various cancer types using tagged antibodies." Clinical Cancer Research 18.23 (2012): 6436-6445.

[23] Minutolo, Nicholas G., et al. "Quantitative control of gene-engineered T-cell activity through the covalent attachment of targeting ligands to a universal immune receptor." Journal of the American Chemical Society 142.14 (2020): 6554-6568.

[24] Cho, Jang Hwan, James J. Collins, and Wilson W. Wong. "Universal chimeric antigen receptors for multiplexed and logical control of T cell responses." Cell 173.6 (2018): 1426-1438.

[25] Landgraf, Kyle E., et al. "convertible CARs: A chimeric antigen receptor system for flexible control of activity and antigen targeting." Communications biology 3.1 (2020): 1-13.

[26] Lohmueller, Jason, et al. "Post-translational covalent assembly of CAR and synNotch receptors for programmable antigen targeting." bioRxiv (2020).

[27] Lajoie, Marc J., et al. "Designed protein logic to target cells with precise combinations of surface antigens." Science 369.6511 (2020): 1637-1643. 
[28] Cartellieri, M., et al. "Switching CAR T cells on and off: a novel modular platform for retargeting of T cells to AML blasts." Blood cancer journal 6.8 (2016): e458-e458.

[29] Rodgers, David T., et al. "Switch-mediated activation and retargeting of CAR-T cells for B-cell malignancies." Proceedings of the National Academy of Sciences 113.4 (2016): E459-E468.

[30] Sutherland, Ashley R., Madeline N. Owens, and C. Ronald Geyer. "Modular Chimeric Antigen Receptor Systems for Universal CAR T Cell Retargeting." International journal of molecular sciences 21.19 (2020): 7222.

[31] Urbanska, Katarzyna, et al. "A universal strategy for adoptive immunotherapy of cancer through use of a novel T-cell antigen receptor." Cancer research 72.7 (2012): 1844-1852.

[32] Minutolo, Nicholas G., et al. "Quantitative control of gene-engineered T-cell activity through the covalent attachment of targeting ligands to a universal immune receptor." Journal of the American Chemical Society 142.14 (2020): 6554-6568.

[33] Kim, Min Soo, et al. "Redirection of genetically engineered CAR-T cells using bifunctional small molecules." Journal of the American Chemical Society 137.8 (2015): 2832-2835.

[34] Lee, Yong $\mathrm{Gu}$, et al. "Use of a single CAR T cell and several bispecific adapters facilitates eradication of multiple antigenically different solid tumors." Cancer research 79.2 (2019): 387-396.

[35] Ma, Jennifer SY, et al. "Versatile strategy for controlling the specificity and activity of engineered T cells." Proceedings of the National Academy of Sciences 113.4 (2016): E450-E458.

[36] Cho, Jang Hwan, James J. Collins, and Wilson W. Wong. "Universal chimeric antigen receptors for multiplexed and logical control of T cell responses." Cell 173.6 (2018): 1426-1438.

[37] Lajoie, Marc J., et al. "Designed protein logic to target cells with precise combinations of surface antigens." Science 369.6511 (2020): 1637-1643.

[38] Qasim, Waseem, et al. "Molecular remission of infant B-ALL after infusion of universal TALEN gene-edited CAR T cells." Science translational medicine 9.374 (2017).

[39] Liu, Xuan, et al. "Split chimeric antigen receptor-modified T cells targeting glypican-3 suppress hepatocellular carcinoma growth with reduced cytokine release." Therapeutic advances in medical oncology 12 (2020): 1758835920910347.

[40] Haruyama, Yukihiro, and Hiroaki Kataoka. "Glypican-3 is a prognostic factor and an immunotherapeutic target in hepatocellular carcinoma." World journal of gastroenterology 22.1 (2016): 275.

[41] Gao, Huiping, et al. "Development of T cells redirected to glypican-3 for the treatment of hepatocellular carcinoma." Clinical Cancer Research 20.24 (2014): 6418-6428.

[42] Zakeri, Bijan, et al. "Peptide tag forming a rapid covalent bond to a protein, through engineering a bacterial adhesin." Proceedings of the National Academy of Sciences 109.12 (2012): E690-E697.

[43] Ehninger, A., et al. "Distribution and levels of cell surface expression of CD33 and CD123 in acute myeloid leukemia." Blood cancer journal 4.6 (2014): e218-e218.

[44] Taussig, David C., et al. "Hematopoietic stem cells express multiple myeloid markers: implications for the origin and targeted therapy of acute myeloid leukemia." Blood 106.13 (2005): 4086-4092.

[45] Wang, Quan-shun, et al. "Treatment of CD33-directed chimeric antigen receptor-modified T cells in one patient with relapsed and refractory acute myeloid leukemia." Molecular therapy 23.1 (2015): 184-191. 